\title{
Comparison of object classification methods in seed stream separation
}

\author{
Andrey V. Vlasov \\ Department of Automatics and Computer Systems \\ Tomsk Polytechnic University \\ Tomsk, Russia \\ andark@tpu.ru
}

\author{
Alexander S. Fadeev \\ Department of Automatics and Computer Systems \\ Tomsk Polytechnic University \\ Tomsk, Russia \\ fas@tpu.ru
}

\begin{abstract}
The paper presents a study of machine learning approaches to detect and classify seeds of a grain crop in order to enhance agricultural seed purification line. The main features of seeds that are hard to recognize during a separation with mechanical methods are resolved with the help of machine learning approach. The main machine learning methods used in research was traditional machine learning and deep learning based on neural networks. A special training image database was retrieved in order to check if the stated approaches are reasonable to use and develop. A set of tests is provided to show the effectiveness of the machine learning applied to solve the stated problem.
\end{abstract}

Keywords-image processing, seeds sorting, classification, feature extraction, convolutional neural network, automatic detection, grains, agriculture.

\section{INTRODUCTION}

The present agricultural production cycle covers most of society needs, but growing consumption rate stimulates further development of all agricultural processes. The purification of seed material becomes a significant part in this development and needs to be improved. The traditional mechanical sorting approaches to purify the seed material are cheap and widely spread but cannot provide enough precision and make the output pure enough. This problem can be solved with the help of the power of computing facilities. In order to enhance the efficiency of sorting stage different computer methods are combined together and usually applied in final iterations of the purification process.

Today object detection and classification methods are performed with the help of computer vision $(\mathrm{CV})$. These methods can be successfully applied to solve the most problems of object detection, tracking and classifying. The CV technologies usually increase the efficiency of systems, reduce time of processing and automate routine work [14]. The processing optimization with $\mathrm{CV}$ is promising from different points of view $[3,6,11]$. Nevertheless, seed classification and seed sorting approaches are not well developed the studies are not held profoundly.

This paper presents an investigation and research of an image processing approach that can provide efficient seed classification precision and recognition speed for the grain crops purification and sorting stages.

\section{MATERIALS AND METHODS}

Five kinds of grain crop seeds were used as a testing material in image caption and further processing. These five kinds were chosen as massively used and sold on Russian market and these seeds are valuable for agriculture sphere. There are: wheat, rapeseed, phacelia, flax, white mustard seeds. Each kind represents a group of objects to classify. According to several free public image training datasets (The University of Texas, Computer science department's datasets: http://www.cs.utexas.edu/ grauman/courses/spring2008/datase ts.htm; The University of Edinburgh, the School of Informatics' datasets: http://homepages.inf.ed.ac.uk/rbf/CVonline/Imagedbase.htm; the University of Illinois, datasets for Computer Vision Research: http://www-cvr.ai.uiuc.edu/ponce_grp/data/) there are no suitable seed image databases for the stated problem available for the experiments. Thus, real seeds were used to fill a training image dataset. The experimental dataset was filled with captured images of these five seed kinds. Each kind contains about a hundred of images, thus the whole set is about five hundred images in total. The Fig. 1 show photos of real seeds that were used to fill a small training dataset. The equipment used were a cheap Canon digital camera and stripes of white LED light along with a small amount of seeds. This equipment provided a minimum cost experimental data for estimation purposes to prove the further research necessity.

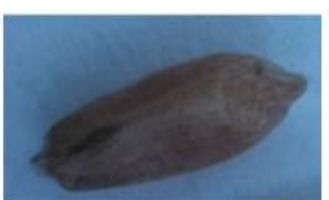

a

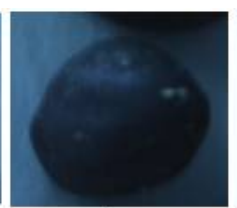

b

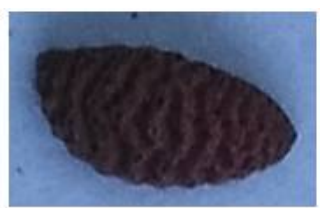

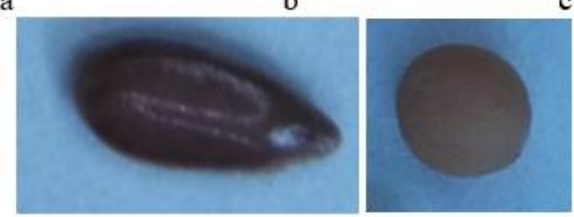

Fig. 1. Seed image examples from training dataset: $a-$ wheat; $b$ - rapeseed; $c$ - phacelia; d - flaxseed; e - white mustard seed

A small dataset allows to estimate the efficiency of different methods and provide some basic experimental results without huge investments. This approach is risks preventive and was chosen because the necessary equipment is expensive and the processing can result with low accuracy.

In order to conduct research of an object recognition and classification for the stated problem the computer vision technologies and rapidly developed method, such as machine learning, were used [2,9]. These methods are available in the Matlab toolboxes (the versions used are R2016b and R2017a). The first is traditional machine learning which is based on common feature extraction techniques include Histogram of Oriented Gradients (HOG), Speeded Up Robust Features (SURF), Local Binary Patterns (LBP), Haar wavelets, and color histograms $[8,9]$. The second method is based on deep learning with convolutional neural networks $(\mathrm{CNN})$. Both methods resulted in a classifier to detect the kind of seed in the image and were tested to compare precision $[5,11]$. 


\section{CLASSIFICATORS COMPARISON}

Reasoning from theoretical premises, object classification in images can be done by using different approaches, but the most appropriate for the stated problem is a computer vision. The CV is a relatively new technology and the existing studies are not fully cover the technology's potential. Nevertheless, it has already proven itself as a working solution for real problems.

The stated problem requires to develop a classifier based on $\mathrm{CV}$ using some of the available approaches and compare them to find the best one. The first step was to extract the most significant parameters and choose what methods to use.

As it is seen from Fig. 1, seeds have different visual features: shape, color, surface, glance. These parameters are hardly recognizable with traditional mechanical methods and separation is made basically for size and weight. Applying a camera with fast computer vision processing with predefined classifier can cover the limitations of mechanical sorting and will provide the better sorting accuracy $[6,7]$.

\section{A. Traditional machine learning}

According to the stated problem, one of the most significant requirements is a processing speed. Regarding to the task, seeds will fall down in front of the camera and a chosen method should provide fast image capture and object classification to send a signal to remove a bad seed. Thus the problem is to find out what kind of grain crop is detected to separate it from the main seed flow. The next parameter is precision. Precision is needed to provide a high level classification to minimize grain crop recognition error and reduce the number of separating iterations to reach the desirably clean product.

The stated problem with specified requirements can be solved by using a traditional machine learning approach [2]. The method is based on image feature extraction, descriptors retrieval, clustering and completing of visual words vocabulary (Fig. 2).

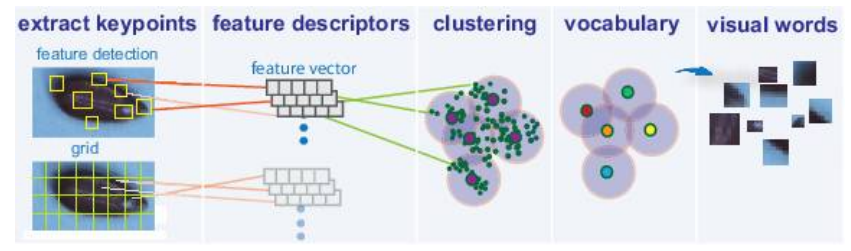

Fig. 2. A seed image bag of features and visual words extraction

This approach can be applied because it doesn't require a huge number of training images to prepare a classifier. Applying this classifier, the result is provided with a set of values that shows how close is the object to each class (Fig. 3). The most value indicates the closest predictable class of the object. The speed of classifying is high enough to use it in a real-time system.

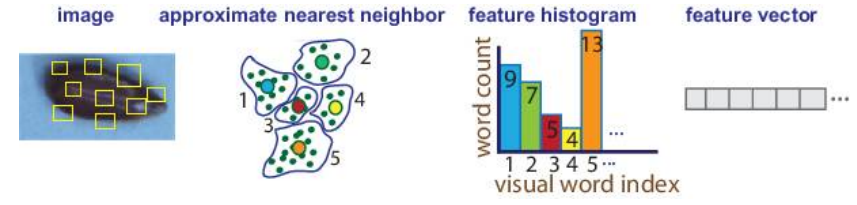

Fig. 3. A seed image traditional machine learning classification process

\section{B. Deep learning}

Meanwhile the traditional machine learning approach is not the only possibility to classify seeds images and improve separation process. The latest research in this field based on deep neural networks have achieved great success in terms of processing accuracy and computational performance for images [13]. The approach based on convolutional neural networks $(\mathrm{CNN})$ becomes widely spread due to its efficiency [12].

The main idea of the method is to automatically extract features directly from image data into convoluted inner layers of network, subsample feature set and continue this processes until it is possible to form the output classified values (Fig. 4). The advantage of deep learning is that pixels of the image are allowed as input neurons of the first layer without special preprocessing. The last layer will have as much neurons as a number of target classes. Thus it is perfect to classify an input image according to the stated problem.

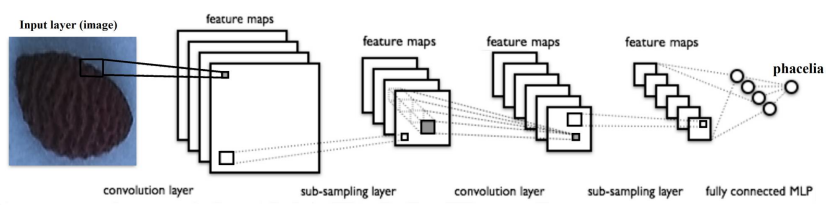

Fig. 4. Typical architecture of convolutional neural network

The most noticeable difference between traditional and deep learning is that deep learning provides more accuracy but at the same time needs to be trained on much bigger and variable image dataset. In comparison the deep learning approach is evaluated by MATLAB up to $95 \%$ classification accuracy when traditional learning is only about $75 \%[8,10]$.

A deep learning training supposed to base on thousands of images for each class. The training process of deep learning is performance and memory consuming $[1,10]$. This problem is partly solved by using video cards for parallel computations, but still the bottle neck for deep learning is memory: the single video card can provide only up to $12 \mathrm{~GB}$ on top models. Nevertheless, this approach theoretically meant to be more efficient and will provide best results for the stated task.

\section{RESUlTS}

The experiments with seed classifier trainings were conducted using MATLAB with the Computer Vision toolbox and the Neural Network toolbox along with the prepared database of seed images. There were two stages in testing.

\section{A. Experiments part 1}

The first test stage was about traditional machine learning. The Computer Vision toolbox was used to train a classifier. The default MATLAB parameters for this method were adjusted to reach better results according to previous research 
[15]. The vocabulary size of 5000 words was chosen for the test. Now the experiment contained the following steps:

- Load prepared seed images dataset.

- Randomly divide the dataset into training and test parts with $0.9 / 0.1$ ratio.

- Extract a bag of features.

- Train image category classifier on the training part of the dataset.

- Evaluate the accuracy on the test part of the dataset.

The average classification accuracy according to 10 individual test iterations for traditional machine learning was $95.49 \%$. The result is good and even higher than theoretical average value and also it shows that this classifier can be used to process small seed images.

\section{B. Experiments part 2}

The second test stage was about deep learning. The Neural Network toolbox was used to train a classifier with deep learning. Deep learning in MATLAB is recommended to use with the help of a CUDA-capable NVIDIA ${ }^{\text {TM }}$ GPU with compute capability 3.0 or higher. The GeForce GTX 1070 was used to process images and train the classifier. The procedure of this experiment contained very similar steps as the first one:

- Randomly divide image set into training and test parts with $0.9 / 0.1$ ratio.

- Train a classifier with a CNN.

- Evaluate the accuracy on the test part of the dataset.

The experiment has the same approach with 10 individual test iterations. The average classification accuracy for deep learning was $98,76 \%$ where individual test reached even $100 \%$ accuracy. This result is very good, but it only shows that this method is appropriate to use with this kind of objects in images.

\section{CONCLUSION}

To sum up the studies of grain crop's seed classification with computer vision it is possible to conclude that the computer vision technologies are appropriate for improving the process of seed classification in order to provide better grain crop purification processing. The machine learning approach is recommended as flexible and precise way to solve the stated problem and enhance an existing mechanical seed sorting.

The experiments showed accurate classification of different seed categories and approved that computer vision can be used to classify seed color, shape, surface and glance - features that were previously hard to separate using only mechanical methods.

According to the experiments results the traditional machine learning can be used to train a classifier even with a small amount of training data but in comparison with deep learning it has less accuracy. Also traditional machine learning is not that flexible and expendable to add new categories as it can be done with CNNs. Other categories of grain crop seeds such as rice, oat and corn will be added to extend the variety of training images, so deep learning has an advantage in this area.

The deep learning approach is expected to have the best results if it will be used with vastly more images to train. It is possible to achieve by making a prototype of a sorting machine with a high speed camera. This will allow to capture as much images in real conditions as needed to train a good classifying CNN. Thus the next research will be focused on making this prototype and retrieving an appropriate image database to create a classifier.

\section{REFERENCES}

[1] I. Arel, D. C. Rose, and T. P. Karnowski, "Deep Machine Learning - A New Frontier in Artificial Intelligence Research [Research Frontier]," IEEE Comput. Intell. Mag., Vol. 5, no. 4, pp. 13-18, 2010

[2] C. M. Bishop, Pattern Recognition and Machine Learning, vol. 4, no. 4. Springer, 2006

[3] F. Esposito and D. Malerba, "Machine learning in computer vision," Appl. Artif. Intell., vol. 15, pp. 693-705, 2001

[4] Y. Fang, T. Chang, R. Zhai, and X. Wang, "Automatic recognition of rape seeding emergence stage based on computer vision technology," 2014 3rd Int. Conf. Agro-Geoinformatics, Agro-Geoinformatics 2014, 2014

[5] F. N. Jawad Nagi, Syed Khaleel Ahmed, "A MATLAB based Face Recognition System using Image Processing and Neural Networks," 4th Int. Colloqium Signal Process. its Appl., pp. 83-88, 2008

[6] K. Kiratiratanapruk and W. Sinthupinyo, "Color and texture for corn seed classification by machine vision,” 2011 Int. Symp. Intell. Signal Process. Commun. Syst. "The Decad. Intell. Green Signal Process. Commun. ISPACS 2011, pp. 7-11, 2011

[7] J. Li, G. Liao, and F. Xiao, "Rapeseed seeds colour recognition by machine vision," Proc. 27th Chinese Control Conf. CCC, pp. 146-149, 2008

[8] MATLAB and Computer Vision Toolbox Release 2016a, The MathWorks, Inc., Natick, Massachusetts, United States.

[9] MathWorks, (2016). Computer Vision Toolbox: User's Guide (R2016a). Retrieved August 18, 2016 from http://www.mathworks.com/discovery/feature-extraction.html

[10] K. Prasad, D. C. Nigam, A. Lakhotiya, and D. Umre, "Character Recognition Using Matlab's Neural Network Toolbox," International Journal of u- and e- Service, Science and Technology Vol. 6, No. 1, February, 2013. 2013

[11] J. Schmidhuber, "Deep Learning in neural networks: An overview," Neural Networks, vol. 61. Elsevier Ltd, pp. 85-117, 2015

[12] V. Singh and S. Mohan Rao, "Application of image processing and radial basis neural network techniques for ore sorting and ore classification," Miner. Eng., vol. 18, no. 15, pp. 1412-1420, 2005

[13] W.-T. S. I. and V. S.-R. U. an E. S.-P. C. N. N. Shi, J. Caballero, F. Huszár, J. Totz, A. P. Aitken, R. Bishop, D. Rueckert, and Z. Wang, "Real-Time Single Image and Video Super-Resolution Using an Efficient Sub-Pixel Convolutional Neural Network," in CVPR, 2016

[14] I. V. Tsapko, A. V. Vlasov "Object's border and position allocating in an X-ray image", Applied Mechanics and Materials. 2015, Vol. 756, pp. $667-672$

[15] A. V. Vlasov, A. S. Fadeev "A machine learning approach for grain crop's seed classification in purifying separation", Journal of Physics: Conference Series. - 2017. — Vol. 803 : Information Technologies in Business and Industry (ITBI2016) : International Conference, 21-26 September 2016, Tomsk, Russian Federation : [proceedings]. [012177, 6 p.]. 